IFUP-TH 7/98, SNBC/98-02-01

hep-th/9802050

\title{
ABELIANIZATION OF SU(N) GAUGE THEORY WITH GAUGE INVARIANT DYNAMICAL VARIABLES AND MAGNETIC MONOPOLES
}

\author{
Adriano Di Giacomof and Manu Mathur? \\ 2 Dipartimento di Fisica dell' Universitâ and I.N.F.N, \\ Piazza Torricelli 2 Pisa, 56100 Italy \\ ${ }^{3}$ S. N. Bose National Centre for Basic Sciences, \\ JD Block, Sector 3, Salt Lake City, Calcutta 700091, India.
}

\begin{abstract}
It is shown that $\mathrm{SU}(\mathrm{N})$ gauge theory coupled to adjoint Higgs can be explicitly re-written in terms of $\mathrm{SU}(\mathrm{N})$ gauge invariant dynamical variables with local physical interactions. The resultant theory has a novel compact abelian $U(1)^{(N-1)}$ gauge invariance. The above abelian gauge invariance is related to the adjoint Higgs field and not to the gauge group $\mathrm{SU}(\mathrm{N})$. In this abelianized version the magnetic monopoles carrying the magnetic charges of $(N-1)$ types have a natural origin and therefore appear explicitly in the partition function as Dirac monopoles along with their strings. The gauge invariant electric and magnetic charges with respect to $U(1)^{(N-1)}$ gauge groups are shown to be vectors in root and co-root lattices of $\mathrm{SU}(\mathrm{N})$ respectively. Therefore, the Dirac quantization condition corresponds to SU(N) Cartan matrix elements being integers. We also study the effect of the $\theta$ term in the abelian version of the theory.
\end{abstract}

\footnotetext{
${ }^{1}$ Partially supported by EC Contract ERBFMRXCT97-0122 and by MURST

${ }^{2}$ Electronic address: digiacomo@pi.infn.it

${ }^{3}$ Electronic address: manu@boson.bose.res.in, manu@ibmth.difi.unipi.it
} 


\section{INTRODUCTION}

The subject of magnetic monopoles has been fascinating ever since they were proposed by Dirac [1] in 1931 to explain electric charge quantization in abelian theory. He showed that the quantization condition between electric (e) and magnetic (g) charges:

$$
e g=2 \pi n, \quad n=0, \pm 1, \pm 2, \ldots \ldots
$$

was both necessary and sufficient to quantize an abelian theory in the presence of magnetic monopoles. As a consequence of these external magnetic charges, the abelian gauge group becomes compact. On the other hand, in the non-abelian $\mathrm{SU}(\mathrm{N})$ gauge theories the gauge group is defined to be compact independent of any charges. Like the abelian case above, the compactness of the non-abelian gauge group is again intimately related to the presence of magnetic monopoles. However, these magnetic charges are not visible in the original non-abelian Lagrangian but occur as topological excitations of the theory.

Perhaps the most important role of the magnetic monopoles in physics is their expected role in the mechanism of quark confinement. It is widely believed that their condensation could provide an interesting and sufficient framework to explain quark confinement along the lines of dual Meissner effect [2]. This conjucture has been tested explicitly in the context of much simpler compact lattice quantum electrodynamics (CLQED) where the magnetic degrees of freedom manifestly appear in the partition function. In the confining phase of CLQED, these magnetic charges condense in the vacuum leading to a linear potential between the electric charges. It is generally hoped that the confinement of the color charges in the non-abelian theories will have its roots manifest in its abelianised version. If so, the qualitative picture of confinement in the non-abelian theories could be very similar to the confining phase of CLQED. Therefore, an important problem before studying the vacuum properties of the non-abelian theories, is to abelianize them so as to make the contribution of the topological magnetic degrees of freedom to the partition function explicit. A similar but simpler problem arises in the context of abelian Higgs theories with Abrikosov-Nielsen-Olesen (ANO) vortices as topological excitations. This problem has been widely studied in the past. In this abelian framework, the radial decomposition of the complex 
Higgs field has been extremely useful in extracting out the contribution of the ANO vortices from the partition function [3]. However, to our knoweldge, a similar construction for the non-abelian gauge theories and its relevence to topological magnetic monopoles and hence to confinement is still lacking inspite of large amount of literature on this subject. This will be the motivation and the subject of this paper. In the non-abelian $\mathrm{SU}(\mathrm{N})$ gauge theories, based on the suggestion of 't Hooft [4], extensive work has been done in the past to study the topological magnetic monopoles in effective abelian theories via "abelian projections". By abelian projection it is meant that the $\mathrm{SU}(\mathrm{N})$ gauge group is gauge fixed such that only its maximal Cartan subgroup $U(1)^{N-1}$ is left untouched. In these approaches with pure gauge theories certain collective excitations of the theory act as an effective "SU(N) adjoint Higg field" and the space time points where two of the eigenvalues are equal are the possible locations of the magnetic charges. However, unlike the abelian Higgs model or the compact lattice quantum electrodynamics, these issues have been largely addressed at the level of kinematics and there has been very little progress at the level of dynamics besides Monte Carlo simulations [5]. In this paper we formulate the idea of radial decomposition in the context of $\mathrm{SU}(\mathrm{N})$ gauge theory and study its consequences to the magnetic monopoles. A very qualitative discussion of its relevance to confinement is given at the end. For the sake of simplicity, we take the adjoint Higgs mentioned above not as composite field but as one of the microscopic field present in the Lagrangian. However, most of the results presented below are general. They depend only upon the adjoint transformation property of the Higgs and not on the detailed form of the Lagrangian except on its gauge invariance. Also, the low energy physics of the pure $\mathrm{SU}(\mathrm{N})$ gauge theory can be thought of as the large mass limit of the Higgs in the present formulation. We find that the non-abelian radial decomposition to be described below has many resemblences with the corresponding abelian formulation along with some novel results. They are are summarised below:

1. $\mathrm{SU}(\mathrm{N})$ gauge theories with adjoint Higgs can be rewritten completely in terms of explicitly gauge invariant fields. This is similar to the abelian Higgs model (see section II),

2. In terms of the above dynamical variables novel compact abelian gauge invariance $U(1)^{N-1}$ (not a subgroup of $S U(N)$ ) naturally emerges, 
3. As a consequence of abelianiasation, the magnetic monopolest in the theory now are point like and their contribution to the partition function can be explicitly extracted. As expected, this contribution is similar to the one proposed by Dirac in the context of abelian gauge theory with external magnetic charges [6]. Moeover, they carry charges of $(N-1)$ types corresponding to the $(N-1) \mathrm{U}(1)$ gauge groups and couple to their corresponding abelian gauge fields with coupling proportional to $\left(\frac{1}{e}\right)$. This feature is again similar to the abelian Higgs model where the radial decomposition makes the contribution of the ANO vortices to the partition function explicit and they appear with inverse coupling [3].

4. The Dirac quantization condition has simple interpretation in terms of the geometrical properties of the roots and co-roots of the $\mathrm{SU}(\mathrm{N})$ group and corresponds to the scalar product of any root and co-root vectors being integers.

The results and the techniques presented below are independent of the space time dimension (d) but we illustrate the idea in $d=4$. The organisation of the article is as follows. The section II is devoted to the kinematical aspects of the SU(N) adjoint Higgs and is independent of any non-abelian gauge invariance of a theory. It starts with the description of the basic idea of constructing $\mathrm{SU}(\mathrm{N})$ adjoint Higgs dynamics in the "body fixed frame" (BFF) along with its novelties. This idea is partly borrowed from the rigid body classical dynamics where the angular motion of the rigid body is described in terms of the angular velocities of the BFF. After implementing the radial decomposition of Higgs field, we will find the angular velocity description useful in constructing the $\mathrm{SU}(\mathrm{N})$ gauge invariant variables. The section IIB is devoted to describe the inbuilt $U(1)^{(N-1)}$ abelian gauge invariance in the above frame. It is emphasized that this local abelian invariance is solely due to the description of the adjoint Higgs in terms of its angular velocities in the

\footnotetext{
${ }^{4}$ These monopoles include both $\mathrm{SU}(\mathrm{N})$ t' Hooft Polyakov types which are solutions of the classical equations of motions in the Higgs phase as well as the ones which are not the solutions and occur independent of the phases. The latter can be looked upon as local defects in space time and might also contribute to the dynamics and the spectrum of the theory, e.g in the case of pure Q.C.D their condensation can lead to confinement of the colored gluons. The classical equations of motion or their solutions will not be used anywhere in this paper.
} 
BFF. Hence, the above abelian invariance should not be confused with any subgroups of the initial SU(N) gauge group. Partly for this reason, we have introduced the $\mathrm{SU}(\mathrm{N})$ gauge group and the corresponding gauge fields only in the section III after describing the $U(1)^{(N-1)}$ abelian gauge invariance in detail. We must emphasize here that in the full gauge theory in the unitary gauge with the Higgs oriented in a particular fixed direction, we recover all the results of t' Hooft [4]. In this sense what follows can be looked upon as the explicit gauge invariant formulation of t' Hoofts ideas at the level of partition function. In what follows, we will also be borrowing heavily from the work of t' Hoofts. In section II.C we describe the geometrical constraints on the angular velocities of the body fixed frame of any $\mathrm{SU}(\mathrm{N})$ adjoint Higgs field. Singularities in these constraints will eventually lead to topological magnetic charges in the theory. One important assumption through out this paper is that the singularities in the constraints in the section II.C occur at discrete space time points. The section II.D involves the computation of the Jacobians on going to the angular co-ordinates and then to the angular velocity descriptions. In section III we include the gauge fields. In this section, exploiting the $\mathrm{SU}(\mathrm{N})$ gauge transformation properties of the Higgs angular velocities and of the gauge fields we construct the $\mathrm{SU}(\mathrm{N})$ gauge invariant and $U(1)^{(N-1)}$ gauge covariant fields $Z_{\mu}^{ \pm}(x)$ along with $\mathrm{SU}(\mathrm{N})$ gauge invariant $(N-1)$ photons of $U(1)^{(N-1)}$ gauge group. All the features discussed above are general and model independent. Only at the end of the section III we will introduce the SU(N) Higgs model with an arbitrary gauge invariant potential to implement these ideas explicitly at the level of the partition function. Therefore, these techniques can also be applied to pure $\mathrm{SU}(\mathrm{N})$ Yang Mills theory where there is no microscopic Higgs field present in the Lagrangian. The role of Higgs is now played by a particular choice of composite gluonic field. The section IV is devoted to study of the magnetic monopoles which are necessarily the locations where the the descripition of the Higgs field in terms of its angular velocities fails. At the end we summarize our results and discuss qualitatively a probable relevance of the radial decomposition to the problem of confinement. 


\section{THE BODY FIXED FRAME, ANGULAR VELOCITIES AND THE $U(1)^{(N-1)}$ GAUGE INVARIANCE}

We first describe our notations and some definitions which will be useful to describe $\mathrm{SU}(\mathrm{N})$ gauge theory and the corresponding magnetic monopoles within a general framework. A hermitian traceless $N \times N$ matrix $\phi$ is described by $\left(N^{2}-1\right)$ real parameters. The set of all such matrices will form

the real vector space $R^{N^{2}-1}$. Any hermitian traceless matrix $\phi$ will be called a vector in $R^{N^{2}-1}$. In a particular basis, $\phi$ will also be denoted by $\vec{\phi}$ where each of its $N^{2}-1$ elements being the components in that basis. The scalar product of two vectors $\phi_{1}$ and $\phi_{2}$ is defined by

$$
\left(\phi_{1}, \phi_{2}\right) \equiv \frac{1}{2} \operatorname{Tr}\left(\phi_{1} \phi_{2}\right) .
$$

Here $\operatorname{Tr}$ stands for the trace. The norm of a vector $\phi$ is $(\phi, \phi)$. The space $R^{\left(N^{2}-1\right)}$ can be spanned by any set $\left(N^{2}-1\right)$ orthonormal (with respect to the scalar product (2)) basis vectors.

In the Cartan Weyl basis they are constructed such that $H_{i},(i=1,2 \ldots \ldots,(N-1))$ are the set of commuting elements and $E_{ \pm \alpha}, \alpha=1, \ldots \ldots, \frac{\left(N^{2}-N\right)}{2}$ are the noncommuting ladder operators In the standard notations [7] their algebra is:

$$
\begin{aligned}
{\left[H_{i}, H_{j}\right] } & =0 \\
{\left[H_{i}, E_{\alpha}\right] } & =K_{i}(\alpha) E_{\alpha} \\
{\left[E_{\alpha}, E_{\beta}\right] } & =\vec{K}(\alpha) \cdot \vec{H} \quad \text { if } \quad \alpha=-\beta \\
& =N_{\alpha, \beta}^{\gamma} E_{\gamma} \quad \text { otherwise. }
\end{aligned}
$$

In (3) the $(N-1)$ dimensional root correspoding to the ladder operator $E_{\alpha}$ is denoted by $\vec{K}(\alpha) . N_{\alpha, \beta}^{\gamma}$ are constants depending upon the group and are non-zero only if $\vec{K}(\alpha)+\vec{K}(\beta)=\vec{K}(\gamma)$. We will denote the Cartan subspace by $\mathrm{H}$ and the $N-1$ simple roots spanning $\mathrm{H}$ will be denoted by $\vec{K}\left(\alpha_{s}\right), s=1,2, \ldots, N-1$. The elements of the algebra (3) are normalised such that $\left(H_{i}, H_{j}\right)=\delta_{i j},\left(E_{\alpha}, E_{\beta}\right)=(1 / 2) \delta_{\alpha+\beta, 0}$. From now onwards this 
basis will be called the "Space fixed frame" (SFF) because it is given in terms of a set of constant (space time independent) elements. A convenient realisation of this basis, to be used often hereafter, is defined in terms of the $N^{2}$ matrices $e_{a b},(\mathrm{a}, \mathrm{b}=1,2 \ldots ., \mathrm{N})$ with matrix elements $\left(e_{a b}\right)_{c d} \equiv \delta_{a c} \delta_{b d}$. In terms of these matrices

$$
\begin{gathered}
H_{i}=A_{i}\left[\sum_{j=1}^{i} e_{j j}-i e_{i+1 i+1}\right], \\
E_{+\alpha}=e_{a b}, \quad E_{-\alpha}=e_{b a} \quad a<b .
\end{gathered}
$$

In the representation (幽 $A_{i} \equiv \sqrt{\frac{2}{i(i+1)}}$ are the normalisation constants and the root vectors are labelled by $\vec{K}_{a b}$. Another representation to be used is the $\mathrm{SU}(\mathrm{N})$ Hermitian Gell-Mann $\lambda$ matrices: $\lambda_{i} \equiv H_{i}, \lambda_{+\alpha} \equiv E_{+\alpha}+E_{-\alpha}$ and $\lambda_{-\alpha} \equiv i\left(E_{+\alpha}-E_{-\alpha}\right)$ satisfying $\left(\lambda_{a}, \lambda_{b}\right)=\delta_{a b},\left(a=1,2 \ldots \ldots N^{2}-1\right)$ 门.

Given a vector $\phi$, we will now define a $\left(N^{2}-1\right)$ dimensional orthonormal "body fixed frame" (BFF) as follows: We first note that $\phi(x)$ being a hermitian traceless matrix, it can be unitarily transformed into the $(N-1)$ dimensional Cartan sub-basis:

$$
U(x) \phi(x) U^{-1}(x) \equiv \sum_{h=1}^{N-1} \rho_{(h)} \lambda_{h} .
$$

Here $\mathrm{U}(\mathrm{x})$ is a $\mathrm{SU}(\mathrm{N})$ matrix in the coset space $\frac{S U(N)}{U(1)^{N-1}}$. Further the diagonalisation procedure (5) is unique upto the $N$ ! arrangements of the $\mathrm{N}$ eigenvalues $\left(v_{1}, v_{2} \ldots, v_{N}\right)$ of $\phi(x)$ along the diagonal. This permutation of the $\mathrm{N}$ eigenvalues form a permutation group of order $N$ ! which is isomorphic to the the Weyl reflection group [7] of the SU(N). Explicitly, the operator which interchanges the the eigenvalue $v_{a}$ with $v_{b}$ (田) is

$$
\omega_{[a, b]}=\operatorname{expi} \frac{\pi}{2}\left(E_{+\alpha}+E_{-\alpha}\right) .
$$

\footnotetext{
${ }^{5}$ In what follows the indices h,i,j.. will take values from 1 to $N-1$ and the Greek indices $\alpha, \beta$ will vary from 1 to $\frac{N^{2}-N}{2}$. The indeces a,b,c.. will vary from 1 to $\mathrm{N}$ as well as from 1 to $N^{2}-1$ which will be clear from the context or will be explicitly mentioned.
} 
The exchange operator $\omega_{[a, b]}$ in (66) also corresponds to the reflction across the hyperplane perpedicular to the root $\vec{K}(\alpha)$. Each of the $N$ ! choices of arranging the eigenvalues $v_{a}$ in (5) correspond to choosing the set $\rho_{h}$ in one of the $N$ ! Weyl chambers one for each element of the Weyl group. A Weyl chamber $\mathcal{C}_{\omega}$ corresponding to the Weyl group element $w$ is defined through the $(N-1)$ simple roots as follows:

$$
\mathcal{C}_{\omega}=\left(h \in H,\left(\omega h \omega^{-1}, K\left(\alpha_{s}\right)\right)>0, s=1,2, \ldots, N-1\right) .
$$

$\mathcal{C}_{\omega=1}$ is called the fundamental Weyl chamber (FWC). The hyperplanes seperating the Weyl chambers are called the Weyl walls. A vector with two of the eigenvalues equalf lies on one of the Weyl walls. Using these discrete Weyl reflections in $\mathrm{U}(\mathrm{x})$, it is always possible to choose $\rho_{(h)} \geq 0$. In the basis (田), the choice with all the eigenvalues arranged in the decreasing order of magnitude from top to bottom corresponds to $\rho_{h} \geq 0, \forall h$. This can be seen by the relation:

$$
v_{a}-v_{b}=\vec{\rho} \cdot \vec{K}_{a, b}
$$

for $b=a+1,(a=1,2, \ldots . N-1)$ and using the $\vec{K}$ in the basis (4). This particular Weyl chamber with $\rho_{h} \geq 0, \forall h$ is characterised by the Weyl group element $\omega$ :

$$
\begin{aligned}
\omega & =\omega_{[N, 2]} \omega_{[N-1,3]} \cdots \cdots \cdots \omega_{\left[\frac{N}{2}+2, \frac{N}{2}\right]}, & & N: \text { even } \\
& =\omega_{[N, 2]} \omega_{[N-1,3]} \cdots \cdots \cdots . . \omega_{\left[\frac{(N-1)}{2}+2, \frac{(N-1)}{2}+1\right]}, & & N: \text { odd }
\end{aligned}
$$

In the following the chamber (9) will be called the positive Weyl chamber (PWC). In the case of $\mathrm{SU}(2) \omega$ in (9) is an unit operator and $\mathrm{FWC}$ is also the PWC: a positive real line. However, for $\mathrm{SU}(\mathrm{N}), N>2$, the $(N-1)$ positive $\rho_{i}$ are not completely independent. The eqn. (8) implies the following $N-1$ inequalities defining the PWC:

\footnotetext{
${ }^{6} \mathrm{In} \mathrm{SU}(\mathrm{N})$ gauge theories with $\phi(x)$ as the adjoint Higgs, this is a necessary condition for the magnetic monopoles [4].
} 


$$
\rho_{i} \geq \sqrt{\frac{i-1}{i+1}} \rho_{i-1}
$$

Having thus obtained the $(N-1)$ "radii" for a Hermitian traceless matrix $\phi(x)$, we now define a set of $\left(N^{2}-1\right)$ orthonormal basis vectors $\xi^{a}(x)$ through the "angular" degrees of freedom of $\phi(x)$ :

$$
\xi^{a}(x) \equiv U^{-1}(x) \lambda^{a} U(x) .
$$

The $N^{2}-1$ vectors in (11) form another orthonormal basis in $R^{N^{2}-1}$ which is space time dependent. The components of the BFF axis in the space fixed frame $\left(\lambda_{A}\right)$ are given by $\xi_{A}^{a}=\left(\lambda_{A}, \xi^{a}\right)$ and are real. In the sequel, the components of an arbitrary vector $v$ along the $\xi^{ \pm \alpha}$ will be denoted by $v^{\mp \alpha}$ and will be called the "chiral components" of $v$. The equation (5) defines $\phi(x)$ in terms of $\mathrm{U}$ and the radial variables $\rho_{i} \geq 0$ and is the non-abelian analogue of breaking a complex abelian Higgs field into its radial and angular parts. All the topological properties of $\phi(x)$ are now contained in the unitary matrix $\mathrm{U}(\mathrm{x})$. As mentioned before the abelian analogue of (5) has been widely applied in abelian theories to study topological excitations:

1. In the case of 2-dimensional abelian Higgs model it corresponds to writing the complex Higgs field $\phi(x) \equiv \rho(x) \operatorname{expi\theta }(x)$. If $A_{\mu}(x)$ is the $\mathrm{U}(1)$ gauge field and $\mathrm{e}$ is the electric charge then defining $\omega_{\mu}(x) \equiv$ $\partial_{\mu} \theta(x)$, one can rewrite the theory in terms of the $\mathrm{U}(1)$ gauge invariant variables $e Z_{\mu}(x) \equiv A_{\mu}(x)-\omega_{\mu}(x)$ and the radial field $\rho(x)$. The abelian field strength tensor in terms of $Z_{\mu}$ is $F_{\mu \nu}(Z)=\partial_{\mu} Z_{\nu}(x)-\partial_{\nu} Z_{\mu}(x)-$ $1 / e\left(\partial_{\mu} \partial_{\nu}-\partial_{\nu} \partial_{\mu}\right) \theta^{\text {sing }}$. Here $\theta^{\text {sing }}$ is the multivalued part of the phase angle $\theta$ of $\phi$. The last term in the field strength tensor above describes the ANO vortices.

2. In 4 dimensions the radial decomposition of spin 0 magnetic charges $\phi(x) \equiv \rho(x) \operatorname{expi\theta }(x)$ was exploited to write down a manifestly Lorentz co-variant and local quantum field theory of these particles interacting with electric charges [8]. In this work once again the topological magnetic currents in the final theory were intimately related to the global angular behaviour of $\theta(x)$. 
Motivated by the above results in the context of abelian theories, we now formulate the idea of radial decomposition further in the context of nonabelian gauge theories and exploit it to study the magnetic monopoles. The treatment will be very similar in spirit to that of the ANO vortices in the case of abelian Higgs model [3, 14]. Its final consequences are already summarised in the section I.

In the next section we will express all the color vectors and their space time derivatives in the BFF basis. Being an orthonormal basis we know that it can only undergo rotations in space time. In other words, analogous to rigid body dynamics, these changes will be given in terms of the angular velocities of the BFF. By taking the space time derivative of (11) it is easy to see

$$
\partial_{\mu} \xi^{a}(x)-i\left[\omega_{\mu}(x), \xi^{a}(x)\right] \equiv D_{\mu}(\omega(x))_{a b} \xi^{b}=0
$$

In (12), $\omega_{\mu}(x) \equiv i U^{-1}(x) \partial_{\mu} U(x)$ are the angular velocities of the BFF and $D_{\mu}(\omega)$ are the "covariant" derivatives with respect to the matter angular velocities. The meaning of covariant will be clear after we study the transformation properties of $\xi^{a}(x)$ and $\omega_{\mu}^{a}(x)$ under the abelian (section II.B) and the $\mathrm{SU}(\mathrm{N})$ gauge symmetries (section III).

\section{II.B THE ABELIAN GAUGE INVARIANCE}

The Higgs field in (5) depends only on $\xi^{h}(\mathrm{~h}=1,2, \ldots . .,(N-1))$ and the corresponding radial fields $\rho_{h}(x)$. Therefore, the BFF basis defined in (11) is determined only upto local $U(1)^{(N-1)}$ abelian gauge invariances corresponding to the rotations around each of $\xi^{h}(x)$. This implies that any dynamics rewritten in the BFF will have an inbuilt local $U(1)^{(N-1)}$ invariance independent of any gauge group. This invariance is also compact. Defining

$U^{(H)}(x) \equiv U^{-1}(x)\left(\exp i \sum_{h=1}^{N-1} \theta^{h}(x) \lambda^{h}\right) U(x)$, the local abelian invariances are:

$$
\xi^{a}(x) \rightarrow U^{(H)}(x) \xi^{a}(x) U^{(H)^{-1}}(x)
$$


The computation of the transformation laws of the BFF (11) is straight forward. We denote the $(N-1)$ angles of the compact $U(1)^{N-1}$ gauge group by $\vec{\theta}(x) \equiv\left(\theta^{1}(x), \theta^{2}(x), \ldots \theta^{N-1}(x)\right)$. The $U(1)^{(N-1)}$ abelian gauge transformations of the $\mathrm{BFF}$ basis vectors are given by:

$$
\begin{aligned}
\xi^{h}(x) & \rightarrow \xi^{h}(x) \\
\xi_{ \pm \alpha}(x) & \rightarrow \exp \left(-i \vec{K}_{ \pm \alpha} \cdot \vec{\theta}(x)\right) \quad \xi_{ \pm \alpha}(x) .
\end{aligned}
$$

The vectors $\vec{K}_{ \pm \alpha} \equiv\left(K_{ \pm \alpha}^{1}, K_{ \pm \alpha}^{2}, \ldots \ldots \ldots, K_{ \pm \alpha}^{N-1}\right)$ are the root vectors defined in (3). The abelian transformations (14) induce the following transformations on the Cartan and the chiral components of the angular velocities:

$$
\begin{aligned}
\omega_{\mu}^{h}(x) & \rightarrow \omega_{\mu}^{h}(x)+\partial_{\mu} \theta^{h}(x) \\
\omega_{\mu}^{ \pm \alpha}(x) & \rightarrow \exp \left(i \vec{K}_{ \pm \alpha} \cdot \vec{\theta}(x)\right) \quad \omega_{\mu}^{ \pm \alpha}(x) .
\end{aligned}
$$

Note that the BFF components of the angular velocities in the Cartan subspace transform like abelian gauge fields while its chiral components transform like matter fields with charges proportional to the corresponding root vectors. Once again we emphasize that the abelian symmetries (14) and (15) must not be taken as the subgroups of the gauge group $\mathrm{SU}(\mathrm{N})$ to be introduced later in the section III. Infact, any Higgs model, where the Higgs field is a vector in the internal space, rewritten in the BFF in terms of its angular velocities will have (14) and (15) as its local invariance. A simple example is the $\sigma$ model with global $\mathrm{O}(3)$ invariance. This model in its angular velocity description has a local $\mathrm{U}(1)$ invariance [9].

\section{II.C THE GEOMETRICAL CONSTRAINTS}

In the section III, we would like to describe the dynamics of the adjoint Higgs vector not by its orientation $\mathrm{U}(\mathrm{x})$ in (5) but by its angular velocities. This, as will be shown in the section III will enable us to construct $\mathrm{SU}(\mathrm{N})$ gauge dynamics in terms of explicit gauge invariant variables. However, the 
angular velocity description introduces extra variables. To begin with, we had the Higgs field with the $N-1$ radial fields and the $N^{2}-N$ co-ordinates of the diagonalisation matrix $\mathrm{U}$ in the coset space $S U(N) / U(1)^{N-1}$ describing the orientation of the BFF with respect to the SFF. However, the angular velocities $\omega_{\mu}^{a}(x)$ have $4 \times\left(N^{2}-1\right)$ degrees of freedom. Therefore, in terms of them the dynamics is highly constrained. Naively, these constraints on the angular velocities can be easily computed through its defining equation (12):

$$
\left[D_{\mu}(\omega), D_{\nu}(\omega)\right] \xi^{a}(x) \equiv 0 \quad=>\quad F_{\mu \nu}^{a}(\omega)=0 .
$$

In deriving the constraint $F_{\mu \nu}^{a}(\omega)=0$ in (16) we have assumed that $\left(\partial_{\mu} \partial_{\nu}-\partial_{\nu} \partial_{\mu}\right) \xi^{a}(x) \equiv 0$. This is not true as the BFF basis vectors in general can be functions of multivalued fields in space time. Given a generic Higgs vector we would like to locate the space time points where the right hand side of the constraint (16) is different from zero. Towards this end, we characterize the diagonalization matrix $\mathrm{U}(\mathrm{x})$ by a set of $\left(N^{2}-1\right)$ parameters denoted by $\vec{\Theta}(x)$. The defining equation of $\omega_{\mu}(x)$ in (12) now can be rewritten as

$$
\omega_{\mu}^{a}=-H_{b}^{a}(\vec{\Theta}(x)) \partial_{\mu} \Theta^{b}(x) .
$$

The eqn. (17) expresses the SFF components of the angular velocities in terms of the matrix $H_{b}^{a}(\vec{\Theta}(x))$ which is defined through the composition functions $F\left[\vec{\Theta}_{1} ; \vec{\Theta}_{2}\right]$, (i.e $\left.U\left(\vec{\Theta}_{1}\right) U\left(\vec{\Theta}_{2}\right)\right) \equiv U\left(F\left[\vec{\Theta}_{1} ; \vec{\Theta}_{2}\right]\right)$ of the $\operatorname{SU}(\mathrm{N})$ group [10] as follows:

$$
\begin{aligned}
& \left.M_{b}^{a}(\vec{\Theta}(x)) \equiv \frac{\partial F^{a}[\vec{\Phi} ; \vec{\Theta}(x)]}{\partial \Phi^{b}}\right|_{L i m \vec{\Phi} \rightarrow 0} \\
& M_{b}^{a}(\vec{\Theta}(x)) H_{c}^{b}(\vec{\Theta}(x)) \equiv \delta_{c}^{a} .
\end{aligned}
$$

The equation (17) defining the angular velocities is a straight forward generalization of the angular velocities defined in the case of rigid body classical dynamics [10] to field theory. Now computing the field strength tensor for the angular velocities we find that

$$
\begin{aligned}
\mathcal{F}_{\mu \nu}^{a}(\omega) & =\left[H_{b, c}^{a}(\vec{\Theta}(x))-H_{c, b}^{a}(\vec{\Theta}(x))+f^{a e f} H_{c}^{e}(\vec{\Theta}) H_{b}^{f}(\vec{\Theta})\right] \partial_{\mu} \Theta^{c}(x) \partial_{\nu} \Theta^{b}(x) \\
& +H_{b}^{a}(\vec{\Theta}(x))\left(\partial_{\mu} \partial_{\nu}-\partial_{\nu} \partial_{\mu}\right) \Theta^{b}(x)
\end{aligned}
$$


In (19) $H_{b, c}^{a}(\vec{\Theta})$ is the partial derivative of $H_{b}^{a}(\vec{\Theta})$ with respect to $\Theta^{c}$. However, because of the associativity property of the group, the first term in the square bracket is zero. This term being zero is just the integrability condition of the partial differential equation satisfied by the composition function $F\left[\Theta_{1} ; \Theta_{2}\right]$ due to associativity property of the group. Note that different coordinate systems chosen to describe the unitary matrix will have their own set of structure constants and composition functions but they will all satisfy the above integrability condition. For more details the reader is referred to [10]. The necessary condition for the right hand side of (19) being not equal to zero at some space time point $x_{0}$ is that atleast one of the parameters describing the unitary matrix $U$ should be multivalued function of space time. The sufficient condition is that the support $H_{B}^{A}\left(x_{0}\right) \neq 0$. In what follows, we will assume that the above singular points $x_{0}$ are discretely and not continuosly distributed in the space time. To incorporate these multivalued fields in the theory it is convenient to devide $\Theta(x) \equiv \Theta^{[r]}(x)+\Theta^{[s]}(x)$. Here $\Theta^{[r]}(x)$ and $\Theta^{[s]}(x)$ are single and multivalued functions of space time and are defined by:

$$
\begin{aligned}
& \oint_{\mathcal{C} \in \Delta \Sigma_{\mu \nu}\left(x_{\mu}^{0}\right)} \partial_{\mu} \Theta^{[s]}(x) d x_{\mu}=2 \pi \mathcal{Z}, \quad \mathcal{Z}: \text { Integers } \\
& \oint_{\mathcal{C} \in \Delta \Sigma_{\mu \nu}\left(x_{\mu}\right)} \partial_{\mu} \Theta^{[r]}(x) d x_{\mu} \equiv 0 \quad \forall x_{\mu} \in R^{4} .
\end{aligned}
$$

Here $\mathcal{C}$ is a curve enclosing any surface $\Delta \Sigma_{\mu \nu}\left(x_{\mu}^{0}\right)$ around a singular space time point $x_{\mu}^{0}$. So finally we see that a careful computation of the field strength tensor of the matter angular velocities gives?:

$$
\mathcal{F}_{\mu \nu}^{a,(n p)}(\omega(x))=H_{b}^{a}(\vec{\Theta}(x))\left(\partial_{\mu} \partial_{\nu}-\partial_{\nu} \partial_{\mu}\right) \Theta^{[s] b}(x) .
$$

We have attached an extra superscript $(\mathrm{np})$ on $\mathcal{F}_{\mu \nu}^{a}(\omega)$ now and it stands for "non-perturbative". Its origin will be clear when we discuss the full gauge theory. In section IV we will explicitly study the singular nature of (21) and its consequences to the complete partition function of the gauge theory and in particular to magnetic monopoles.

\footnotetext{
${ }^{7}$ This computation also shows that the unitary transformation defined in (5) must not be confused with the gauge transformation (see section III) and trivially absorbed in the gauge invariant measure.
} 


\section{II.D THE MEASURE}

Before including the gauge fields, it is instructive to compute the Jacobian on going from the Euclidean description of the Higgs to the radial and the angular velocity description. After including the gauge fields in the section III and IV we will see that through this Jacobian we are led to $(N-1)$ types of Dirac terms describing the magnetic monopoles in the final abelian version of the $\mathrm{SU}(\mathrm{N})$ theory. In the first step of this section we will compute the Jacobian from the Eucledian $(\phi)$ to the angular and the radial co-ordinates defined in (5). In the second step we will further transform the angular part of this measure into the measure in terms of the angular velocities. Some of the results in the first part of this section are already in the literature [11]. However, for the sake of completeness and also because our interpretation of some of the terms in the final measure is different (see footnote 8), we will reproduce them briefly below.

The metric tensor in the Euclidean co-ordinates of the adjoint Higgs is defined by $(d \phi(x), d \phi(x))=\delta^{a b} \phi_{a} \phi_{b}$. To compute the measure it is convinient to characterize the diagonalisation matrix $U(\Theta)$ in terms of the variables in the Cartan subalgebra $\mathrm{H}$ and the coset space $\frac{G}{H}$ :

$$
U(\Theta(\theta, z)) \equiv U_{H}(\theta) U_{\frac{G}{H}}(z) .
$$

Here $\left[\theta_{i}(x), i=1,2 \ldots, N-1\right]$ and $\left[z_{\alpha}(x), \alpha=1,2, \ldots N^{2}-N\right]$ are the variables characterizing the subgroup $U(1)^{N-1}$ and the coset space $S U(N) / U(1)^{N-1}$. Defining $d \omega(z) \equiv i U_{\frac{G}{H}}(z)^{-1} d U_{\frac{G}{H}}(z)=-\lambda^{\alpha}(z) H_{\alpha \beta}(z) \delta z^{\beta}(x)-\lambda^{i}(z) \tilde{H}_{i \beta}(z) \delta z^{\beta}(x)$, $\left[\rho_{i} \lambda^{i}(x), \lambda^{\alpha}(x)\right] \equiv \omega_{\alpha \beta}(\rho) \lambda^{\beta}$ with $\omega(\rho)$ being a linear matrix in the radial variable $\rho(x)$. Now using (5) a straightforward calculation leads to [11]:

$$
\begin{aligned}
& (d \phi, d \phi)=d \rho_{i} d \rho_{j}\left(\lambda^{i}, \lambda^{j}\right)+\left(\left[\rho_{i} \lambda^{i}, d \omega\right],\left[\rho_{i} \lambda^{i}, d \omega\right]\right) \\
& =\sum_{i=1}^{N-1} d \rho_{i}^{2}+\delta z^{\alpha}\left(H^{T}(z) \omega(\rho) \omega(\rho) H(z)\right)_{\alpha \beta} \delta z^{\beta} .
\end{aligned}
$$

In (22) $H^{T}(z)$ stands for the transpose of the matrix $\mathrm{H}$ defined by (17) in the coset space. Thus, the metric $g_{a b}$ in the new co-ordinates is block diagonal: a unit matrix in the Cartan subspace and is determined by the composition 
functions and the structure constants in the coset space. The corresponding Jacobian $J(\rho, z)$ on going to angular variables is $|H(z)||\omega(\rho)|(|A| \equiv \operatorname{det} \mathrm{A})$. In the Cartan basis [11], $|\omega(\rho)|=\prod_{\alpha=1}^{\frac{N^{2}-N}{2}}(\vec{\rho} \cdot \vec{K}(\alpha))^{2}$, where the product over $\alpha$ runs only over the positive roots. Therefore, the Jacobian $J(\rho, z)$ splits into the following radial and angular parts:

$$
J(\rho, z) \equiv J(\rho) J(z) ; \quad J(\rho)=\prod_{\alpha=1}^{\frac{N^{2}-N}{2}}(\vec{\rho} \cdot \vec{K}(\alpha))^{2}, \quad J(z)=|H(z)|
$$

In (23) $\vec{K}(\alpha)$ are the positive roots. Therefore, we finally get?:

$$
\prod_{a=1}^{N^{2}-1} \int d \phi^{a}(x)=\left[\prod_{i=1}^{N-1} \int^{\prime} d \rho^{i}(x)\right] \prod_{\alpha=1}^{\frac{N^{2}-N}{2}}(\vec{\rho} \cdot \vec{K}(\alpha))^{2}\left[\prod_{\alpha=1}^{N^{2}-N} \int d z^{\alpha}\right]|H(z)| .
$$

In the first set of integrations in (24) $(\prime)$ is used to denote the contrained range of the radial integrations due to (10). We would like to convert the last two factors of the measure in (24) into the $\mathrm{SU}(\mathrm{N})$ invariant Haar measure over $\Theta(x)$, i.e.,

$$
\int \mathcal{D} U(\Theta) \equiv \prod_{a=1}^{N^{2}-1} \int d \Theta^{a}|H(\Theta)|=\prod_{i=1}^{N-1} \int d \theta^{i} \prod_{\alpha=1}^{N^{2}-N} d z^{\alpha}|H(\Theta(\theta, z))| .
$$

This can be seen by exploting the $U(1)^{N-1}$ gauge invariance of the starting Euclidean measure. We make the $U(1)^{N-1}$ gauge transformation $z \rightarrow \Theta(\theta, z)$ and introduce an unity of the form, $\prod_{i=1}^{N-1}\left(1 / 2 \pi \int d \theta_{i}\right) \equiv 1$ in (24). Thus we get the manifest $\mathrm{SU}(\mathrm{N})$ invariant measure:

$$
\prod_{a=1}^{N^{2}-1} \int d \phi^{a}(x)=\left[\prod_{i=1}^{N-1} \int^{\prime} d \rho^{i}(x)\right] \prod_{\alpha=1}^{\frac{N^{2}-N}{2}}(\vec{\rho} \cdot \vec{K}(\alpha))^{2} \int \mathcal{D} U(\Theta) .
$$

\footnotetext{
${ }^{8}$ In [1] the integration over the radial variables $\rho_{h}(x)$ is restricted to the fundamental Weyl chamber of SU(N) and therefore has non-trivial boundaries. Moreover, the integration over $\mathrm{z}$ in (24) is treated as trivial and ignored taking it as the gauge group volume in the full adjoint Higgs gauge theory.
} 
In the second step, to go to the angular velocity description, we introduce an unity in the form of a $\delta$ function over the angular velocities and then integrate over the true gauge degrees of freedom $\Theta^{[r]}(x)$ :

$$
\begin{gathered}
\int d \vec{\omega}_{\mu}^{a}(x) \delta\left(\vec{\omega}_{\mu}^{a}(x)-\left(\xi^{(a)}, U(\Theta) \partial_{\mu} U^{-1}(\Theta)\right)\right) \equiv 1 \\
\int \mathcal{D} U(\Theta) \delta\left(\omega_{\mu}^{a}-\left(\xi^{a}, U(\Theta) \partial_{\mu} U^{-1}(\Theta)\right)=\int \mathcal{D} \Theta^{[s]} \delta\left(F_{\mu \nu}^{a}(\vec{\omega})+\mathcal{F}_{\mu \nu}^{a(n p)}\left(\Theta^{[s]}\right)\right) .\right.
\end{gathered}
$$

For the sake of simplicity we have omitted to indicate the products over space time points, Lorentz and $\mathrm{SU}(\mathrm{N})$ group indeces in (26). The first equation in (26) is just the definition of the angular velocities. The right hand side of the second equation is motivated by the $\mathrm{SU}(\mathrm{N})$ invariance of the Haar measure of both left and right hand side. Thus, in terms of the radial fields and the angular velocities:

$$
\begin{aligned}
\prod_{a=1}^{N^{2}-1} \int d \phi^{a}(x)= & {\left[\prod_{i=1}^{N-1} \int^{\prime} d \rho^{i}(x) \prod_{\alpha=1}^{\frac{N^{2}-N}{2}}(\vec{\rho} . \vec{K}(\alpha))^{2}\right] } \\
& \int d \omega_{\mu}^{a}(x) \int \mathcal{D} \Theta^{[s]} \delta\left(F_{\mu \nu}^{a}(\vec{\omega})+\mathcal{F}_{\mu \nu}^{a(n p)}\left(\Theta^{[s]}\right)\right) .
\end{aligned}
$$

We will see the relevance of (27) later after including the gauge fields in the section III and in the context of the magnetic monopoles in the section IV.

\section{THE SU(N) GAUGE GROUP}

Till now our discussion has been independent of any gauge group and the corresponding gauge fields. At this stage we intoduce the $\mathrm{SU}(\mathrm{N})$ gauge group acting on the adjoint Higgs $\phi(x)$ and gluonic fields $W_{\mu}(x)$ as:

$$
\begin{aligned}
\phi(x) & \rightarrow G(x) \phi(x) G^{-1}(x) \\
W_{\mu}(x) & \rightarrow G(x) W_{\mu}(x) G^{-1}(x)+i G(x) \partial_{\mu} G^{-1}(x) .
\end{aligned}
$$


Before introducing any particular model, it is useful to study the $\mathrm{SU}(\mathrm{N})$ gauge transformation properties of the new variables $\xi^{a}$ and $\omega_{\mu}(x)$ we had introduced in the Section II. From the gauge transformation of the adjoint Higgs and the equation (5) it is clear that all $\xi^{h}$ also transform like adjoint fields. If $\mathrm{G}(\mathrm{x})$ is the gauge transformation matrix in the fundamental representation of $\mathrm{SU}(\mathrm{N})$ then the orthonormality of the BFF basis vectors forces us to define:

$$
\begin{aligned}
\xi^{a}(x) & \rightarrow G(x) \xi^{a}(x) G^{-1}(x) \\
\omega_{\mu}(x) & \rightarrow G(x) \omega_{\mu}(x) G^{-1}(x)+i G(x) \partial_{\mu} G^{-1}(x)
\end{aligned}
$$

It is easy to see from (14) that under $U(1)^{(N-1)}$ local gauge transformations the components of $W_{\mu}(x)$ in the $\operatorname{BFF}\left(W_{\mu}^{a}(x) \equiv\left(W_{\mu}, \xi^{a}\right)\right.$ undergo the following induced transformations:

$$
\begin{aligned}
W_{\mu}^{h}(x) & \rightarrow W_{\mu}^{h}(x), \\
W_{\mu}^{ \pm \alpha}(x) & \rightarrow \exp \left(i \vec{K}^{ \pm \alpha} \cdot \vec{\theta}(x)\right) W_{\mu}^{ \pm \alpha}(x) .
\end{aligned}
$$

We now define the covariant gauge fields by

$$
e Z_{\mu}(x) \equiv \omega_{\mu}(x)-W_{\mu}(x)
$$

In (31) we have explicitly introduced a constant e for later convenience. It will eventually be identified with the $\mathrm{SU}(\mathrm{N})$ coupling constant. As the BFF basis vectors $\xi^{a}(x)$ and $Z_{\mu}(x)$ both transform covariantly under SU(N) gauge transformation (see (28), (29) and (31)), the components of the vector $Z_{\mu}(x)$ in the $\mathrm{BFF} Z_{\mu}^{a}(x)\left(\equiv\left(Z_{\mu}, \xi^{a}(x)\right)\right.$ are explicitly $\mathrm{SU}(\mathrm{N})$ gauge invariant. Therefore, any specific SU(N) gauge model with adjoint Higgs (which may be a composite field) rewritten in terms of $Z_{\mu}^{a}(x)$ variables will be explicitly gauge invariant. On the other hand, under $(U(1))^{(N-1)}$ gauge transformations (see (15) and (30)):

$$
\begin{aligned}
Z_{\mu}^{ \pm \alpha}(x) & \rightarrow \exp \left(i \vec{K}_{ \pm \alpha} \cdot \vec{\theta}\right) Z_{\mu}^{ \pm \alpha}(x) \\
Z_{\mu}^{h}(x) & \rightarrow Z_{\mu}^{h}(x)+\frac{1}{e} \partial_{\mu} \theta^{h}(x) .
\end{aligned}
$$


We notice that under $(U(1))^{(N-1)}$ the $(N-1)$ components of $Z_{\mu}$ within the Cartan subalgebra of the BFF transform like abelian gauge fields. Following the language of t' Hooft, they will be called "photons" and will be denoted by $A_{\mu}^{h}(x)\left(\equiv Z_{\mu}^{h}(x)\right)$. They are of $N-1$ types and explicitly $\mathrm{SU}(\mathrm{N})$ gauge invariant. Further, the $\mathrm{SU}(\mathrm{N})$ gauge invariant chiral components $Z_{\mu}^{ \pm \alpha}(x)$ transform like abelian matter fields with charges proportional to their root vectors. These gauge invariant electric charges we compactly denote by a set of $N^{2}-N$ vectors $\vec{Q}_{[ \pm \alpha]}$, each of them with $N-1$ components:

$$
\vec{Q}_{[ \pm \alpha]}=e \vec{K}( \pm \alpha) .
$$

We can now define their abelian co-variant derivatives (to be used later) by

$$
D_{\mu}(A) Z_{\nu}^{ \pm \alpha}(x) \equiv\left(\partial_{\mu}-\vec{A}_{\mu}(x) \cdot \vec{Q}_{[ \pm \alpha]}\right) Z_{\nu}^{ \pm \alpha}(x)
$$

Having described the general idea and the framework we now introduce the $\mathrm{SU}(\mathrm{N})$ Higgs model and explicitly show its abelianization and as a consequence the emergence of $(N-1)$ types of magnetic monopoles in the partition function. The Lagrangian is:

$$
\mathcal{L}=-\frac{1}{4 e^{2}}\left(F_{\mu \nu}(W), F_{\mu \nu}(W)\right)-\frac{1}{2}\left(D_{\mu} \phi(x), D_{\mu} \phi(x)\right)+V(\phi(x)) .
$$

In (35) $\phi$ and $W_{\mu}$ are the hermitian traceless matrices describing the Higgs and the gluon fields respectively. $D_{\mu} \phi \equiv \partial_{\mu} \phi(x)-i\left[W_{\mu}, \phi\right]$ and $F_{\mu \nu}(W) \equiv \partial_{\mu} W_{\nu}-\partial_{\nu} W_{\mu}-i\left[W_{\mu}, W_{\nu}\right]$. In (35) e is the $\mathrm{SU}(\mathrm{N})$ coupling constant introduced in (31). The Higgs potential can be any $\mathrm{SU}(\mathrm{N})$ gauge invariant function of the Higgs field and will not play any crucial role in what follows.

At this stage we would like to trade off the Higgs field by its angular velocities and re-express (35) completely in terms of the $\omega_{\mu}(x)$ and the gluonic fields $W_{\mu}(x)$. Using the $\delta$ functions in the measure (26) over the matter angular velocities, it is easy to see that the matter and the field strength tensor parts of the Lagrangian are given by: 


$$
\begin{gathered}
\left(D_{\mu}(W) \phi(x), D_{\mu}(W) \phi(x)\right)=e^{2} \sum_{\alpha=1}^{\frac{\left(N^{2}-N\right)}{2}}\left(\sum_{h=1}^{(N-1)} \rho_{(h)} K_{+\alpha}^{(h)}\right)^{2} Z_{\mu}^{+\alpha} Z_{\mu}^{-\alpha} \\
+\sum_{h=1}^{N-1}\left(\partial_{\mu} \rho_{(h)}\right)^{2} . \\
\frac{(F, F)}{e^{2}}=\sum_{\alpha=1}^{\frac{\left(N^{2}-N\right)}{2}}\left(D_{\mu}(A) Z_{\nu}^{+\alpha}-D_{\nu}(A) Z_{\mu}^{+\alpha}-e \sum_{\gamma, \delta=1}^{\frac{\left(N^{2}-N\right)}{2}} N_{\gamma, \delta}^{\alpha} Z_{\mu}^{+\gamma} Z_{\nu}^{+\delta}+\frac{1}{e} \mathcal{F}_{\mu \nu}^{\alpha,(n p)}\right) \\
+\sum_{h=1}^{(N-1)}\left(\partial_{\mu} A_{\nu}^{h}-\partial_{\nu} A_{\mu}^{h}-\frac{i}{2} \sum_{\alpha=1}^{\frac{\left(N^{2}-N\right)}{2}} Q_{[+\alpha]}^{h}\left(Z_{\mu}^{+\alpha} Z_{\nu}^{-\alpha}-Z_{\nu}^{+\alpha} Z_{\mu}^{-\alpha}\right)+\frac{1}{e} \mathcal{F}_{\mu \nu}^{h,(n p)}\right)^{2} .
\end{gathered}
$$

In (37) $N_{\gamma, \delta}^{\alpha}$ are the constants defined in (3) and are non-zero iff $\vec{K}(+\gamma)+$ $\vec{K}(+\delta)=\vec{K}(+\alpha)$. The covariant derivative $D_{\mu}(A)$ are defined in (34). We would like to emphasize the following interesting features of the final lagrangian given by (36,37):

1. The Lagrangian (36) and (37) is not only expressed in terms of explicitly $\mathrm{SU}(\mathrm{N})$ gauge invariant variables and locally but also manifestly invariant under the abelian gauge group $(U(1))^{(N-1)}$, i.e under the transformations (32). Both these results were expected to begin with:

(a) the $U(1)^{N-1}$ invariance as a consequence of describing the dynamics of the Higgs in the BFF in terms of its angular velocities,

(b) The explicit $\mathrm{SU}(\mathrm{N})$ gauge invariance due to the fact that the components of a vector in the $\mathrm{BFF}^{a}(x)$ are explicitly gauge invariant.

\footnotetext{
${ }^{9}$ We are ignoring the term $\left.\mathcal{F}^{a,(n} p\right)$ describing the magnetic monopoles with their unphysical (non-local and non-gauge invariant) Dirac strings. This will be discussed in the Section IV.
} 
2. Note that in (37) both (e) and $\left(\frac{1}{e}\right)$ appear. Therefore, $\mathcal{F}^{a,(n p)}$ is a nonperturbative term. In the next section we will show that it describes the $(N-1)$ magnetic monopole of the $\mathrm{SU}(\mathrm{N})$ theory.

The final partition function is easy to compute. The action (36) and (37) has only implicit dependence on $\omega_{\mu}^{a}(x)$ through $Z_{\mu}^{a}(x)$. Therefore, trading off the integration over the gauge fields $W_{\mu}^{a}(x)$ in terms of the explicitly gauge invariant fields $Z_{\mu}^{a}(x)$ in (31), the angular velocity integrations over their delta functions in (26) give rise to unity. This leaves us with a complete gauge invariant description of the $\mathrm{SU}(\mathrm{N})$ adjoint Higgs theory with nonperturbative topological degrees of freedom manifest at the quantum level. In the following sections, we will further discuss the topological aspects of the theory i.e, the configurations having non-zero measure over $\Theta^{[s]}$ in (27) and the associated non-perturbative terms proportional to the inverse $\mathrm{SU}(\mathrm{N})$ coupling constant in (37).

\section{THE MAGNETIC MONOPOLES}

Having abelianized the $\mathrm{SU}(\mathrm{N})$ gauge theory to the $U(1)^{(N-1)}$ gauge group, it is expected that we will encounter magnetic monopoles carrying $(N-1)$ types of topological magnetic charges corresponding to to each of the $\mathrm{U}(1)$ groups. The presence of the magnetic monopoles can already be seen in the non-perturbative terms with couplings proportional to $\frac{1}{e}$ in (37). Note that the starting non-abelian theory (35) in terms of $W_{\mu}(x)$ and $\phi(x)$ had no such "magnetic" term in the lagrangian. In the final $U(1)^{(N-1)}$ abelianised version, it is also expected that the contribution of these magnetic terms to the action will be similar to the one proposed by Dirac in the context of abelian gauge theory with external magnetic charges [6]. We will proceed to show how these results emerge naturally in this section. We first note that the $(N-1)$ Noether currents of the $U(1)^{N-1}$ gauge symmetries are given by:

$$
\left.J_{\mu}^{i}\right|_{[i=1,2, \ldots, N-1]}=i \sum_{\alpha=1}^{\frac{N^{2}-N}{2}} Q_{[\alpha]}^{i}\left[\left(Z_{\nu}^{-\alpha} D_{\mu}(A) Z_{\nu}^{+\alpha}-c . c\right)+\right.
$$




$$
\left.e \sum_{\gamma, \delta=1}^{\frac{N^{2}-N}{2}} N_{\gamma, \delta}^{\alpha}\left(Z_{\mu}^{-\gamma} Z_{\nu}^{-\delta} Z_{\nu}^{+\alpha}-c . c\right)+2 \partial_{\nu}\left(Z_{\mu}^{+\alpha} Z_{\nu}^{-\alpha}-c . c\right)\right] .
$$

We also define the topological currents:

$$
K_{\mu}^{i} \equiv \frac{1}{e} \partial_{\nu} \tilde{\mathcal{F}}_{\mu \nu}^{i,(n p)}
$$

Now the $(N-1)$ abelian field strength tensors:

$$
\left.F_{\mu \nu}^{i}\right|_{[i=1,2, \ldots, N-1]}=\partial_{\mu} A_{\nu}^{i}-\partial_{\nu} A_{\mu}^{i}-\frac{1}{e} \mathcal{F}_{\mu \nu}^{i,(n p)}
$$

satisfy their corresponding "Maxwells Equations" with topological magnetic sources, i.e:

$$
\partial_{\mu} F_{\mu \nu}^{i}=J_{\nu}^{i}, \quad \partial_{\mu} \tilde{F}_{\mu \nu}^{i} \equiv K_{\nu}^{i}
$$

In (39) and (41) $\tilde{F}_{\mu \nu} \equiv \frac{1}{2} \epsilon^{\mu \nu \rho \sigma} F_{\rho \sigma}$. The first set of the equations in (41) are the equations of motion of the $(N-1) A_{\mu}(x)$ abelian gauge fields and the second set contains $(N-1)$ Bianchi identities. The question now is to identify the generic space time points where the topological magnetic currents $K_{\mu}^{i}$ do not vanish, i.e, the world lines of magnetic monopoles. These are the points where the two of the eigenvalues of $\phi(x)$ are equal and the radial decomposition (5) breaks down [四. The eqn. (8) implies that at such points the Higgs field lies on one of the domain walls enclosing the PWC and the angular measure (27) vanishes. In the neighbourhood of these singular points $\left(x_{0}\right)$ the Higgs field lies in one of the $\left(N^{2}-N\right) / 2 \mathrm{SU}(2)$ subgroups of $\mathrm{SU}(\mathrm{N})$ [ be written as:

$$
\phi_{[\alpha]}(x) \equiv \phi\left(x_{0}\right)+|\epsilon(x)|\left[\phi_{[\alpha]}^{h} h_{[\alpha]}+\left(\phi_{[+\alpha]} e_{[-\alpha]}+\phi_{[-\alpha]} e_{[+\alpha]}\right)\right] .
$$


In (42) $\phi\left(x_{0}\right)$ is a constant diagonal matrix which lies on one of the domain walls enclosing PWC, $\epsilon(x)$ is a field $\rightarrow 0$ as $x \rightarrow x_{0}$ and

$$
\begin{gathered}
h_{[\alpha]} \equiv \sum_{i=1}^{N-1} \frac{K^{i}(\alpha) H^{i}}{(\vec{K}(\alpha) \cdot \vec{K}(\alpha))}, e_{[ \pm \alpha]} \equiv \frac{1}{\sqrt{\vec{K}(\alpha) \cdot \vec{K}(\alpha)}} E_{[ \pm \alpha]} \\
\phi_{[\alpha]}^{h}(x)=\cos \tilde{\theta}(x), \quad \phi_{[ \pm \alpha]}(x)=\sin \tilde{\theta}(x) \exp \pm i \tilde{\psi}(x) .
\end{gathered}
$$

In (43) $\vec{K}(\alpha)$ is one of the $N-1 \operatorname{roots}\left(\vec{K}_{1,2}, \vec{K}_{2,3}, \ldots . \vec{K}_{N-1, N}\right)$ enclosing the PWC and $\tilde{\theta}$ and $\tilde{\psi}$ are the polar and azimuthal angles in the $\mathrm{SU}(2)$ subspace corresponding to the root $\vec{K}(\alpha)$. Now the diagonalization matrix $\mathrm{U}$ in (5) can be written as:

$$
U_{[\alpha]}(x)=\exp \left(\left(\frac{e_{[+\alpha]}-e_{[-\alpha]}}{\sqrt{2}}\right) \frac{\tilde{\theta}(x)}{2}\right) \exp \frac{i}{2}\left(h_{[\alpha]} \tilde{\psi}(x)\right) .
$$

The angular velocities for the above $(N-1)$ types of configurations in the $\mathrm{BFF}$ are given by:

$$
\begin{gathered}
\omega_{\mu,[\alpha]}^{\beta} \approx 0 \\
\left.\omega_{\mu,[\alpha]}^{h}\right|_{[h=1,2, \ldots, N-1]}=-\cos (\tilde{\theta})\left(\frac{2 K^{h}(\alpha)}{\vec{K}(\alpha) \cdot \vec{K}(\alpha)}\right) \partial_{\mu} \tilde{\psi}^{\text {sing }}+\text { regular terms } .
\end{gathered}
$$

In (45) $\tilde{\psi}^{\text {sing }}$ is the singular part of $\tilde{\psi}$ in (43) having non-zero support only along the polar axis $(\tilde{\theta}=0 / \pi)$. The symbol $\approx 0$ means zero upto regular terms which do not contribute to $\mathcal{F}_{\mu \nu}^{a,(n p)}$. From (45) the non-perturbative terms describing the magnetic monopoles are

$$
\begin{aligned}
\mathcal{F}_{\mu \nu}^{\alpha,(n p)}\left(\omega_{[\alpha]}\right) & \approx 0 \\
\left.\mathcal{F}_{\mu \nu}^{h,(n p)}\left(\omega_{[\alpha]}\right)\right|_{h=1,2, \ldots \ldots N-1} & =-\hat{K}^{h}(\alpha) \cos \tilde{\theta}(x)\left(\partial_{\mu} \partial_{\nu}-\partial_{\nu} \partial_{\mu}\right) \tilde{\psi}^{\text {sing }}(x) \\
& = \pm \hat{K}^{h}(\alpha)\left(\partial_{\mu} \partial_{\nu}-\partial_{\nu} \partial_{\mu}\right) \tilde{\psi}^{\text {sing }}(x) .
\end{aligned}
$$


Thus, these magnetic charges are proportional to the co-root vectors, $\overrightarrow{\hat{K}}(\alpha) \equiv(2 \vec{K}(\alpha) / \vec{K}(\alpha) \cdot \vec{K}(\alpha))$. The last equation in (46) shows the presence of the Dirac strings along the internal polar axes, $(\theta(x)=0, \pi)$ if the azimuthal field $\psi(x)$ around it has a non-trivial homotopy index $\mathcal{Z}(20)$. These Dirac strings carry singular fluxes of amount $(2 \pi / e) \overrightarrow{\hat{K}}(\alpha) \mathcal{Z}$ towards the points where $\theta$ flips from 0 to $\pi$ (i.e the $\epsilon(x)$ in (42) vanishes and two of the eigenvalues are equal). These are the locations of the Dirac monopoles [ [.] with quantized magnetic charges in the units of $\frac{4 \pi}{e} \overrightarrow{\hat{K}}(\alpha)$. Note that the Dirac strings are not gauge invariant but the world lines of the magnetic monopoles are gauge invariant. The magnetic charge can now be expressed as a set of $N-1$ vectors, $\vec{M}_{[ \pm \alpha]}$, each with $N-1$ components:

$$
\vec{M}_{[ \pm \alpha]}=\frac{4 \pi}{e} \mathcal{Z} \overrightarrow{\hat{K}}( \pm \alpha)
$$

In (47) $\mathcal{Z}$ is the homotopy index of the mapping $S_{\text {physical }}^{1} \rightarrow S_{\text {internal }}^{1}$ provided by the multivalued $\psi^{\text {sing }}$ field. The equations (33) and (47) imply the Dirac quantization condition:

$$
\vec{Q}_{[ \pm \alpha]} \cdot \vec{M}_{[ \pm \beta]}=4 \pi \mathcal{Z}_{[ \pm \alpha, \pm \beta]} ; \quad \forall \alpha, \beta .
$$

Above $\mathcal{Z}_{[ \pm \alpha, \pm \beta]}$ is the set of all integers and we have used the fact that the scalar products of the root and co-root vectors are integers. Expanding the root and co-root vectors in terms of simple roots and co-roots with integer coefficients, the Dirac quantization condition (48) can be viewed as a simple consequence of the fact that the Cartan matrix elements are integers. In [12] a quantization condition similar to (48) was derived for the adjoint Higgs model of any compact simple Lie group but for a very special solution of the classical equations of motion. In [12 the following equations:

$$
D_{\mu} F^{a \mu \nu}(W)=0, \quad D_{\mu} \phi^{a}(x)=0, \quad \frac{\partial V(\phi)}{\partial \phi^{a}}=0
$$

are considered. The solutions of (49) also satisfy the classical equations of motions. In the broken phase where the the $\mathrm{SU}(\mathrm{N})$ group is broken to its 
subgroup T, the equations (49) admit the monopole type solutions satisfying $\partial_{\nu}^{2} W_{\mu}^{a}=0$. The index a takes values in the subgroup $\bar{H}$ which is the Cartan subgroup of $\mathrm{SU}(\mathrm{N})$ conjugated by the unbroken group T. For such special solution, invoking the $\mathrm{Wu}$-Yang formulation the condition (48) was obtained as the condition of single valuedness of the gauge transformations connecting the two vector potentials in the overlapping region.

In the case of $\mathrm{SU}(2), \mathrm{K}=2$ and $\hat{K}=1$ and therefore the magnetic monopoles are quantized in the units of $4 \pi / e$, i.e $g=4 \pi / e \mathcal{Z}$. Moreover, as a special case, the famous $\mathrm{SU}(2)$ t' Hooft Polyakov hedgehog solitonic configurations [17] in the present abelian formulation corresponds to $K_{\mu}^{m a g}=$ $\left(\delta^{3}(\vec{x}), \overrightarrow{0}\right)$ in $(\overrightarrow{39})$ and hence to a point like Dirac monopole located at the origin where $\vec{\phi}(x)=0$ [13. We now discuss the full partition function in the special case of $\mathrm{SU}(2)$. In the case of $\mathrm{SU}(\mathrm{N})$ we have $N-1$ types of monopoles and the procedure trivially generalises except the for the inequalities (10). These inequalities can be accounted for by putting a step function in the radial measure. The integration over $\Theta^{\text {sing }}$ in (27) is the integration over the singular azimuthal angles $\psi(x)$. We will now rewrite it in terms of the Dirac strings. Let $X_{\mu}^{i}\left(\sigma_{1}\right)$ be the gauge invariant world lines of the monopoles and $m^{i}$ a set of integers describing their magnetic charges in the units of $\frac{4 \pi}{e}$. The monopole current is given by $K_{\mu}(x)=\sum_{i=1}^{\infty} m^{i} \int d \sigma_{1} \frac{d X_{\mu}^{i}\left(\sigma_{1}\right)}{d \sigma_{1}} \delta^{4}\left(x-X^{i}\left(\sigma_{1}\right)\right)$. Therefore, the magnetic term in (41) can be written as

$$
\tilde{\mathcal{F}}_{\mu \nu}^{a=3,(n p)}(x)= \pm \frac{4 \pi}{e} \sum_{i=1}^{\infty} m^{i} \int d^{2} \sigma \epsilon^{\alpha \beta}\left(\partial_{\alpha} X_{\mu}^{i}(\sigma) \partial_{\beta} X_{\nu}^{i}(\sigma)\right) \delta^{4}\left(x-X^{i}(\vec{\sigma})\right)
$$

This is exactly the term introduced by Dirac [6] in the context of abelian gauge theory where point particles carrying magnetic charges were put by hand. Noticing that for $\mathrm{SU}(2)$ in (36) and (37) the constants $N_{\alpha, \beta}^{\gamma}$ in (3) are zero, $\mathrm{K}=2$, and $\mathrm{Q}=\mathrm{e}$, we find:

$$
\begin{aligned}
Z & =\sum_{m_{1}, ., m_{\infty}} \prod_{i=1}^{\infty} \int d X_{\mu}^{i}(\vec{\sigma}) J\left(X_{\mu}\right) \int \rho^{2} d \rho \int d Z_{\mu}^{a} \exp -S\left(\rho, \vec{Z}_{\mu}, X_{\mu}^{i}\right) \\
S & =\int\left[\frac{1}{4}\left(F_{\mu \nu}(A)\right)^{2}+\frac{1}{4}\left(D_{\mu}(A) Z_{\nu}^{+}-D_{\nu}(A) Z_{\mu}^{+}\right) \cdot h \cdot c+\frac{i e}{2} F_{\mu \nu} Z_{\mu}^{+} Z_{\nu}^{-}\right. \\
& \left.-\frac{1}{16}\left(Z_{\mu}^{+} Z_{\nu}^{-}-h . c\right)^{2}+\frac{1}{2}\left(e^{2} \rho^{2} Z_{\mu}^{+} Z_{\mu}^{-}+\left(\partial_{\mu} \rho\right)^{2}\right)+V(\rho)\right] d^{4} x
\end{aligned}
$$


Here $\mathrm{J}(\mathrm{X})$ is the Jacobian [14 due to the change of the measure to the string world sheet. The above partition function is manifestly invariant under $\mathrm{U}(1)$ gauge transformations. This is an exact result (with the assumption that the singular points where the Higgs field vanishes are discrete) and no gauge fixing has been done. In the broken phase where $\rho(x)=$ constant + fluctuations, the above partition function describes the interaction of photon with the charged massive spin 1 gauge bosons and magnetic monopoles. The physical fields here are explicitly gauge invariant and have the right electric charges under $U(1)$. It is easy to see that in the unitary gauge $\phi(x)=\sigma_{3}$ which can be chosen only locally, we recover the standard results.

At this stage, having an exact abelian theory with magnetic monopoles in the partition function, we can also convert them into dyons by adding the $\mathrm{CP}$ violating and $\mathrm{SU}(\mathrm{N})$ gauge invariant $\theta$ term [15] in the action (35):

$$
\Delta \mathcal{L}=\frac{e^{2}}{32 \pi^{2}} \theta \sum_{i=1}^{N-1} \epsilon^{\mu \nu \rho \sigma} F_{\mu \nu}^{i}\left(A_{\mu}\right) F_{\rho \sigma}^{i}\left(A_{\mu}\right)
$$

Here $\theta$ is an angle in the range $[0,2 \pi]$. The Maxwells equations (41) are now modified and acquires a $\theta$ dependent term: $\partial_{\mu} F_{\mu \nu}^{i}=J_{\nu}^{i}+\frac{e^{2}}{8 \pi^{2}} \theta K_{\nu}^{i}$. Therefore, all the magnetic charges with strengths $\vec{M}_{[\alpha]}$ also acquire electric charges $\Delta \vec{Q}_{[\alpha]}^{i}=\frac{e}{2 \pi} \theta \vec{M}_{[\alpha]}^{i}$ leading to generalized Schwinger quantization condition. Note that the $\theta$ term added above is not a surface term because of the Dirac strings.

\section{DISCUSSION AND SUMMARY}

Increasing evidence emerges from lattice simulations that dual superconductivity is the mechanism of color confinement, together with a better understanding of the nature of the monopoles involved [16]. No similar results exist, for known reasons, in the coninuum. Any better formulation of the theory can be helpful to progress in that direction. In this work we have shown that the $\mathrm{SU}(\mathrm{N})$ non-abelian analogue of the radial decomposition widely used

in the abelian Higgs theories exists. We find it interesting that many features 
of the abelian theories are also hidden in the non-abelian theories. Some of the outcomes like interpretation of the Dirac quantization condition in terms of elementary group theory results are already emphasised in the text. On the other hand from the point of view of color confinement, probably the most interesting aspects are a) being able to make magnetic monopoles manifest in the partition function, b) the explicit non-abelian gauge invariance of all the fields in the partition function and emergence of the novel compact abelian invariances. The final rewritten partition function has $(N-1)$ photons and the corresponding electrically and magnetically charged fields, all explicitly color neutral. This was the picture proposed by t' Hooft [1] to find the macroscopic variables in Q.C.D.. However, his proposal of abelianization was at kinematical level through gauge fixing with the residual abelian gauge group as the subgroup of the initial gauge group.

As mentioned in the introduction, all types of magnetic monopoles are defined through some particular choice of the effective adjoint Higgs field. All these choices are correlated. Almost all the techniques presented here will go through for all of them. The difference being that the final partition function in (51) will get suitably modified with more complicated form of (36) and the measure. However, the term (37) which contains the magnetic monopole interactions will not change. Thus, having a better control over the magnetic monopoles in the present framework, it is worthwhile to rediscuss qualitatively the t' Hooft-Mandelstam [2] conjucture of confinement via dual Meissener effect. We emphasise that most of the ideas below are taken from [2, 20] and already well known. Our purpose here is essentially to tie them with the present formulation and to the work done in [8]. For simplicity we restrict to the case of $\mathrm{SU}(2)$ and discuss only the issues related to the confining phase.

The question of color confinement is the question of $U(1)^{N-1}$ charge neutrality of the physical spectrum at large distances. The condensation of the magnetic monopoles is a sufficient framework to explain confinement vis dual Meissner effect. Before addressing this question of condensation in the present formulation, a relevant problem is to eliminate the non-local Dirac strings at the level of dynamics. In other words to rewrite the final $U(1)^{N-1}$ gauge theory in a manifestly Lorentz co-variant and manifestly local form. Infact, this problem is very general. It will arise in any abelian theory with magnetic charges. This issue was resolved in [8] in the context of simpler single abelian theory of a spin 0 magnetically charged particle interacting 
with photon and other electrically charged matter. In this work, roughly speaking, the Dirac strings were eliminated from the partition function by the use of an antisymmetric tensor field $H_{\mu \nu}(x)$. In the present context also, probably the integration over the Dirac strings in (51) can be eventually rewritten in terms of an integration over an anti-symmetric tensor field $H_{\mu \nu}$ with an appropriate Jacobian. This can be naively seen by regulating the Dirac string in (40) on a lattice by spreading its flux over a plaquette. The field strength tensor in (40) now corresponds tot?:

$$
F_{\mu \nu}(n)=\Delta_{\mu} A_{\nu}(n)-\Delta_{\nu} A_{\mu}(n)-\frac{4 \pi}{e} H_{\mu \nu}(n)
$$

In (53) $H_{\mu \nu}(n)$ are the integer valued fields on a lattice, $\mathrm{n}$ are the lattice sites and $\Delta_{\mu}$ is the lattice difference operator. The naive continuum limit of (53) reproduces (40). The topological magnetic current in (39) now corrsponds to:

$$
K_{\mu}(n)=\left(\frac{4 \pi}{e}\right) \Delta_{\nu} \tilde{H}_{\mu \nu}(n)
$$

This is also similar to the case of magnetic monopoles in compact quantum electrodynamics on a lattice with Wilson action in its Villain form 18, 19]. As suggested by t' Hooft, one should eliminate all the electric charges, as accurately as possible, by computing light-light-magnetic charge scattering amplitudes and writing down the corresponding effective lagrangian for the photons with magnetic charges [20]. The theory at this stage will be described in terms of the following microscopic degrees of freedoms:

1. The neutral radial degrees of freedom $\rho(n)$ defined in (5) taking values from 0 to $\infty$,

2. Anti-symmetric tensor fields $H_{\mu \nu}(n)$ describing the magnetic charges interacting with photons described by the magnetic vector potentials $A_{\mu}(n)$ (i.e, the magnetic field $\vec{B} \equiv \vec{\nabla} \times \vec{A}$ ).

\footnotetext{
here.

${ }^{10} \mathrm{We}$ are completely ignoring the Jacobian $\mathrm{J}(\mathrm{X})$ in (51) for the qualitative purposes
} 
The monopole creation operators are still non-local (54). Therefore, one should perform a duality transformation at this stage to get a local creation operator for the monopoles. The duality transformation on the photon (in terms of the magnetic vector potential $\left.A_{\mu}(x)\right)$ coupled to $H_{\mu \nu}(x)$ through its topological current and a neutral radial degree of freedom should eventually lead to a complex scalar field $\phi(x)$ minimally coupled to the photons (in terms of electric vector potential $\tilde{A}_{\mu}(x)$, i.e $\left.\vec{E} \equiv \vec{\nabla} \times \overrightarrow{\tilde{A}}\right)$ through its Noether current. This picture was atleast true for a similar but simpler abelian theory [8]. The Noether current above is the current associated with the "dual" U(1) invariance of the $\left(\phi(n), \phi^{*}, \tilde{A}_{\mu}(n)\right)$ system. This final version of the theory will be an effective Landau Ginzberg model of superconductivity in its dual form. The question of color confinement will be the question of the spontaneous symmetry breaking of the dual U(1) invariance. Note that for $\mathrm{SU}(\mathrm{N})$ both $\rho$ and $H_{\mu \nu}$ are $(N-1)$ in number and the dual description should lead to $(N-1)$ types of charged scalar fields. The inequalities (10) for $\mathrm{SU}(\mathrm{N})$ with $N \geq 3$ make it distinct from $\mathrm{SU}(2)$. Its physical consequences, if any, are not clear to us.

Acknowledgement: Manu Mathur acknowledges I.N.F.N. - Italy for the fellowship during which this work was done. It is also a pleasure for M.M to acknowledge Daniele Francesconi, Sara Ongaro and Alvise Santangelo for the pleasant time in Italy. 


\section{References}

[1] P.A.M. Dirac, Proc. Roy. Soc. London A 133 (1931) 60.

[2] G. 't Hooft, High energy physics, ed. A. Zichichi (Editorice Compositori, Bologna, 1976), S. Mandelstam, Phys. Rep. C 23 (1976) 245 .

[3] K. Bardakci, S. Samuel Phys. Rev. D 182849 (1979).

[4] G. 't Hooft Nuc. Phys. B190 [FS3] (1981) 455.

[5] A. S. Kronfeld et. al. Nuc. Phys. B 293 (1987) 461, For a review see: A. Di. Giacomo, LAT 95, Nucl. Phys. B (Proc. Suppl.) 47 (1996) 136, hep-lat/9509036.

[6] P. A. M. Dirac Phys. Rev. 74 (1948) 817.

[7] B. G. Wybourne, Classical Groups for physicists, John Wiley, 1974

[8] M. Mathur, H. S. Sharatchandra, Phys. Rev. Letts. 663097 (1991).

[9] A. D' Adda, M. Lüscher, P. Di Vecchia Phys. Rep. 49 (1979) 239. A. Polyakov Gauge Fields and Strings (Harwood Academic Publishers, 1987).

[10] E. C. G. Sudarshan and N. Mukunda, Classical Dynamics A Modern Perspective, John Wiley and Sons, 1974.

[11] L. V. Prokhorov, S. V. Shabanov, Sov. Phys. Usp. 34 (2) (1991) 108, S. V. Shabanov, Mod. Phys. Lett. A11 (1996) 1081

[12] F. Englert, P. Windey, Phys. Rev. D. 14 (1976) 2728.

[13] Adriano Di Giacomo, M. Mathur, Phys. Letts 400 (1997) 129 hep-th 9701083 .

[14] E. T. Akhmedov et. al. Phys. Rev. D 532087 (1996).

[15] E. Witten Phys. lett. B86 (1979) 283. 
[16] A. Di. Giacomo, "The dual superconductor picture of confinement", hep-th 9710080, to appear in the proceedings of Confinement, Duality and Non-perturbative aspects of Q.C.D., Newton Institute, Cambridge, June 1997, Plenum Press.

[17] G. 't Hooft, Nuc. Phys. B79 (1974) 276, A. M. Polyakov, JETP Lett. 20 (1974) 194.

[18] A. M. Polyakov, Phys. Lett. B59 (1975) 82, A. M. Polyakov Nuc. Phys.B 120 (1977) 429.

[19] T. Banks, R. Myerson and J. Kogut, Nucl. Phys. B 129 (1977) 493

[20] Under the Spell of Gauge Theories, G. t'Hooft, World Scientific Press 